\title{
Influence of Cognitive Abilities, Conative Characteristics and Social Status on Student School Grades in Mother Tongue
}

\author{
Dragan Popovic, Evagelia Boli, Vladimir Savic, Milos Popovic, Milica Bojevic, Marina Kostic
}

Faculty of Sport and Physical Education, University of Pristina temporarily based in Leposavic, Serbia

Email address:

dragan.popovic@pr.ac.rs (D. Popovic)

To cite this article:

Dragan Popovic, Evagelia Boli, Vladimir Savic, Milos Popovic, Milica Bojevic, Marina Kostic. Influence of Cognitive Abilities, Conative Characteristics and Social Status on Student School Grades in Mother Tongue. American Journal of Applied Psychology.

Vol. 4, No. 3, 2015, pp. 55-60. doi: 10.11648/j.ajap.20150403.12

\begin{abstract}
The study was conducted in order to determine the influence of cognitive dimensions, conative characteristics and social status on school grades in the native language of the students of a secondary school of chemical technology. For this purpose, 224 students of a secondary school of chemical technology were included in the sample. To evaluate the effectiveness of the input processor, or perceptual reasoning, IT-1 test was chosen. To evaluate the effectiveness of the serial processor, or symbolic reasoning, AL-4 test was selected. To evaluate the effectiveness of the parallel processor, or identification of relations and correlates, S-1 test was chosen. For the assessment of conative characteristics, CON6 measurement instrument was selected by means of which the following conative regulators were evaluated: regulator of activity, regulator of organic functions, regulator of defense reactions, regulator of attack reactions, system for coordination of regulatory functions, system for integration of regulatory functions and system for excitation and inhibition. To evaluate social status, a model constructed by Saksida and Momirovic was applied. INST2 and questionnaire SSMIN were used in this research. For the evaluation of students' academic achievements, their school grades were used. The academic achievements were checked by means of knowledge tests as indicators of the acquired knowledge and skills. The algorithms and programs implemented within this study are presented in full and, the results of these programs are analyzed.
\end{abstract}

Keywords: Latent, Dimension, Matrix, Vectors, Variance

\section{Introduction}

All attempts to explain human behavior using only personality variables or only by means of situational variables, are unsuccessful because the problem itself is set in a wrong way. The sole reason for a particular behavior lies neither in personality variables nor situational variables taken separately, but in their interrelation, when an individual's activities and performance provide that interaction with a new quality.

This problem can be brought under the philosophical concept of science and nature, as it is shown, exclusively and widely, by Radonjic. Radonjic says: "Nature has proved to be merciless towards scientists. While scientists strive for simplicity and economy in all theories, nature squanders its abundance and diversity. Since nature will not adapt to the standards of science, scientists will have to adapt the abundance and diversity of nature. "

Menchiskaya points out that there are often complaints that experiments and studies involve only the intellectual side of development, not the personality of a student as a whole. She believes that the development of the whole personality, and not just one aspect of it, should always be borne in mind.

Recognizing the above facts, if we really want to study the behavior of an individual, primarily academic success or failure, we must deal with his or her personality in whole. Through psychological research in this direction, we will try to clarify which personality traits of a student lead to success and how they affect each other.

So, on the basis of these and other similar findings, the general methodological framework of research of conditionality of a student's academic success has been extended from environmental variables to personality variables. Therefore, a higher and adequate level of the research approach should provide data on the level of development and a way of organization of those personality traits that are relevant to the student's academic achievement, 
that is, determine the contribution of each trait in the organizational structure of the personality during the educational process.

The knowledge of the contribution of certain personality characteristics and their organizational structures to educative effects allows for establishing eligibility for education, or carrying out particular educational interventions by measuring these traits in students. Today there are a large number of studies, but few scientific findings, on the dependence of educational success on differently organized traits in the personality structure, and if there are any, it could not be argued that the findings are scientific facts or principles and rules, but logical and empirical indications on the level of probability and hypotheticality. The exception is intellectual abilities, motivation and some broader dimensions of personality as well as the social status of a student that will be discussed in the study.

This study is characterized by the complexity of the research subject that will inevitably result in close interweaving of application of various methods and tools for data collection and processing.

\section{Methods}

\subsection{The Sample of Respondents}

Among other things, the number of respondents in the sample depends on the level of statistical inference and the choice of mathematical and statistical models. Based on the chosen statistical-mathematical model and program, goals and tasks, the sample included 224 respondents. In all factor procedures, it should constantly be kept in mind that the analysis results depend on three main systems which determine the selection and transformation of information: the sample of variables, sample of respondents and the chosen extraction, or rotary method.

Based on these criteria and experience from previous studies, the sample of 224 respondents is considered to be sufficient for this research. In defining the population from which the sample was drawn, except for the above, other restrictions or stratification variables won't be applied.

The population from which the sample was drawn for this research consists of I, II, III and IV-grade students of a secondary school of chemical technology.

The sample of students systematically covered four classes in each grade of the school. The selection had to meet the research methodology requirements, especially the size, representativeness and homogeneity, which make it possible to generalize the research results to the entire population with a high probability.

The classes selected for the sample shall consist of students who most likely have the same or similar social status characteristics, as well as classes that are not included in the sample.

The use of such a sample had the following objectives:

The fact is that the problems in students are insufficiently studied in this country and abroad, therefore there is a certain gap in the factual knowledge about the personality structure of students.

The fact that four age groups were covered, allowed for systematic study of the factors which may lead to certain differences in the development of traits depending on the age or reflection of personality development of students of various ages.

\subsection{The Sample of Variables}

\subsubsection{The Sample of Cognitive Variables}

This research has provided unequivocal evidence that the structure of cognitive abilities is of a hierarchical type at the top of which is the general cognitive factor and below are three primary factors of cognitive abilities related to the effectiveness of the perceptual processor (or perceptual reasoning), effectiveness of the parallel processor (or the ability to identify relations and correlates) and effectiveness of the serial processor (or symbolic reasoning).

To evaluate the effectiveness of the input-processor, or perceptual reasoning, IT-1 test was chosen. To evaluate the effectiveness of the serial processor, or symbolic reasoning, AL-4 test was chosen. To evaluate the effectiveness of the parallel processor, or identification of relations and correlates, S-1 test was chosen.

\subsubsection{The Sample of Conative Variables}

For the assessment of cognitive characteristics, CON6 measurement instrument was chosen to evaluate the following conative regulators: activity regulator (EPSILON), regulator of organic functions $(\mathrm{CHI})$, regulator of defense reactions (ALPHA), regulator of attack reactions (SIGMA), system for the coordination of regulatory functions (DELTA) and system for the integration of regulatory functions (ETA).

\subsubsection{The Sample of Social Status Variables}

To evaluate the social status, the model constructed by Saksida and Momirovic was applied. INST2 and questionnaire SSMIN were used in this research.

\subsubsection{Dependent Variable - Students`Academic Success}

To evaluate the students` academic success, school grades were used. Academic achievement was checked by means of knowledge tests as indicators of the acquired knowledge and skills. In the research, the students academic evaluation is presented by the traditional for this country grading system based on numerical grades of 1 to 5 at the end of a school year (final grade) for all the educational-scientific areas:

- Mathematics and logic: Mathematics

- Natural sciences: Physics and Chemistry

- Languages: mother tongue and foreign language

\subsection{Data Processing Methods}

In order to reach satisfactory scientific solutions, the researchers used, in the first place, correct, then adequate, unbiased and comparable procedures which conformed to the nature of the stated problem and allowed for extraction and transformation of the appropriate dimensions, the testing of 
hypotheses about these dimensions, identification of differences, relations, predictions and diagnoses, as well as establishment of regularities within the research subject area.

Taking that into account, procedures, considered to conform to the nature of the problem and to leave no great restrictions on basic information, were selected for the research purposes.

It is not clear who was the first to propose to perform a regression analysis of criterion variables in the space of continuously distributed regressor variables after the transformation of the regressors into Mahalanobis form. This process is formally described in the study by (Hadzigalic, Bogdanovic, Tenjovic \& Wolf. 1994) but fifteen years before that, a program was written in the SS language and called ORTHOREG by Momirovic. It performs univariate or multivariate regression analysis in Mahalanobis space. A similar program of the same name was also implemented in the SAS programming system, but it is limited only to the case when there is only one criterion variable whose position in the regressor space gives, indeed, very limited information.

As regression analysis in Mahalanobis space has some very convenient comparative properties relative to the standard canonical model of multivariate regression analysis, here will be described an algorithm that generates the greatest amount of usable information about the parameters of the model. The algorithm is implemented in a program written in a matrix language and the program performance is shown in some previous studies.

All the data in this study were analyzed at the Multidisciplinary Research Center, Faculty of Sports and Physical Education, University of Pristina, by means of the software system for data processing developed by Popovic, D. (1980), (1993) and Momirović, K. \& Popovic, D. (2003).

Multivariate regression analysis of the criterion variables of $\mathrm{Zc}$ in the space of Mahalanobis variables of $\mathrm{M}$ can be defined as a solution to the problem $\mathrm{Mb}=\mathrm{Zc}+\mathrm{E} \mid \operatorname{trag}(\mathrm{EtE})=$ minimum. As MtM = I, the solution that is easily obtained by differentiating the function trag (EtE) is $\mathrm{b}=\mathrm{MtZc}=$ Rrr-1/2Rrc and the matrix of partial regression coefficients is, in fact, a matrix of ordinary product - moment coefficients of correlations between the regressors transformed into Mahalanobis form and criterion variables. Of course, therefore, the asymptotic variance of coefficients bjp of matrix $\mathrm{b}$ is simply $\operatorname{sjp} 2=(1-$ bjp2 $) 2 \mathrm{n}-1$, and the tests of hypotheses H0jp: bjp* $=0$ are clearly $\quad$ fjp $=$ bjp2((n - 2) $(1$ - bjp2)-1), because under H0jp: bjp* $=0$ variables fjp have Fisher-Snedecor $\mathrm{F}$ distribution with 1 and $\mathrm{n} 2$ degrees of freedom. Regression functions are now defined by the operation $\mathrm{Y}=\mathrm{Mb}$ with a matrix of covariances $\mathrm{G}=\mathrm{YtY}=\mathrm{btb}$ $=$ RcrRrr-1Rrc, so the diagonal elements of the matrix $\mathrm{r} 2=$ (rp2) = diag G are normal coefficients of determination; and since $\mathrm{ZctY}=\mathrm{RcrRrr}-1 \mathrm{Rrc}=\mathrm{G}$, then rp elements of $\mathrm{r}$ matrix are ordinary multiple correlation coefficients, and the tests of hypotheses H0p: $\mathrm{rp}^{*}=0$ are defined by the functions $\mathrm{fp}=$ $(\mathrm{rp} 2(1-\mathrm{rp} 2)-1)((\mathrm{n}-\mathrm{m}-1) \mathrm{m}-1)$, because under H0p: $\mathrm{rp}^{*}=0$ functions fp have Fisher-Snedecor $F$ distribution with $m$ and $\mathrm{nm} 1$ degrees of freedom. As the matrix of residual variables is $\mathrm{E}=\mathrm{Zc}-\mathrm{Mb}$, then $\mathrm{W}=\mathrm{EtE}=\mathrm{Rcc}-\mathrm{G}$ is a matrix of their covariances. For identification of regression functions, their correlations defined by the matrix $\mathrm{C}=\mathrm{r}-1 \mathrm{G} \mathrm{r}-1$, could sometimes be useful, as well as correlations of residual variables defined by the matrix $\mathrm{F}=\mathrm{S}-1 \mathrm{WS}-1$, where $\mathrm{S} 2=$ diag $\mathrm{W}$ is a matrix of variances of residual variables.

The structure of regression factors in the Mahalanobis space is simply $\mathrm{S}=$ MtMbr-1 = br-1, so sjp elements of matrix $\mathrm{S}$ are ordinary product-moment correlation coefficients. Therefore, the asymptotic variance of sjp coefficients of S matrix is xjp2 $=(1-\operatorname{sjp} 2) 2 \mathrm{n}-1$, and the tests of hypotheses H0jp: SJP $*=0$ are defined by the functions fjp $=\operatorname{sjp} 2((n-2)(1-\operatorname{sjp} 2)-1)$, where under H0jp: sjp* $=0$, fjp variables have Fisher-Snedecor $\mathrm{F}$ distribution with 1 and $\mathrm{n} 2$ degrees of freedom. As b is, in fact, a correlation matrix, in the matrix V2 $=\mathrm{b} \bullet \mathrm{b}=(\mathrm{vjp} 2)$, where $\bullet$ is the Hadamard multiplication operator, there will be regressor variance components and criterion variables under this model of regression analysis. If we mark the sum vector of row $g$ with eg and the sum vector of row $m$ with em, the elements of the vector $\mathrm{j} 2=\mathrm{V} 2 \mathrm{eg}$ will be fractions of the variance of each regressor which was involved in the prediction of a set of criterion variables; of course, in the vector $(\mathrm{emtV} 2) \mathrm{t}=$ vec $\mathrm{r} 2$ there will be coefficients of determination, and the elements in the columns of matrix V2 are parts of the variance of each criterion variable which can be attributed to certain regressor variables. In the tables, in the appropriate columns, the following is calculated and shown:

$\mathrm{R}$ - product-moment coefficients between each of the predictive variables and criteria

Partial R - partial correlation coefficients of each predictor variable with the criterion variable

Beta - standardized regression coefficients, i.e. coordinates of the criterion vector projected in the space of predictor variables

$\mathrm{P}$ - percentage of contribution of each predictor variable to the explanation of the variance of the criterion variable

SIGMA B - standard deviation of the partial regression coefficients

Q- likelihood that a beta coefficient will appear, if the actual value of the coefficient is zero.

In the last part of the regression tables, the following is marked with:

DELTA - coefficient of determination, i.e. part of the criterion variance which can be explained by the variance of the predictor variables

RO - coefficient of a multiple correlation between predictor variables

SIG D - standard error of prediction of the criterion variable based on the system of predictor variables

$\mathrm{F}$ - usual F test for testing the significance of the multiple correlation coefficient with df1 and dif 2 degrees of freedom

Sig. - probability of getting a certain value of $F$ relationship, if the actual value of the multiple correlation is zero.

\section{Results}

In studies on applied psychology as well as other anthropological sciences, latent dimensions are generally 
assessed on the basis of the patterns of variables formed within the theoretical models which have been the subject of verification in the previous exploratory or confirmatory analyses of the latent structure of manifest anthropological variables.

The hypothetical latent structure in the research is, therefore, explicitly defined, and hypothetical latent dimensions are covered by a large number of manifest variables whose objects of measurement are known from previous analyses or can be assumed with a high probability on the basis of theoretical models cybernetically formulated in the rule.

In psychology literature, three types of the definition of intelligence are most often mentioned. In behavioristic circles, intelligence is usually identified with "learning capacity", or the ability to acquire new knowledge. Less common is the identification of intelligence with "the ability of abstract thinking." Particular attention is given to the definition of intelligence as "the ability to adapt to new situations." It is rather common in animal psychology. This, of course, does not mean the adaptation in terms of tolerance to exogenous factors, nor to adjustment in the clinical sense.

As academic success depends on a number of factors, it is important to have reliable indicators on which dimensions influence the achievement of maximum results and to what extent. Conative space is the part of personality which is responsible for the modalities of human behavior. Since there are normal and pathological modelities of behavior, analogously, there are normal and pathological conative factors.

The characteristic of normal conative factors is that they are mostly independent of each other and normally distributed in the population. There are few attempts to research the normal modalities of behavior and normal conative factors, so that subspace of personality is not clearly enough defined.

Pathological conative factors are much better defined than normal conative factors in previous studies, and in most cases there are certain theoretical explanations for them.

Pathological conative factors are thought to be responsible for those types of behavior which reduce the adaptive level of humans considering their potential possibilities. The impact of conative factors on different activities is not the same. There are activities that are less susceptible to the influence of conative factors, and there are those on which the influence of these factors is crucial. This influence can be positive or negative depending on the type of factors and activities. So, there is no activity that would be completely independent of the influence of conative factors.

The source of most problems associated with examining the social status as a research subject or control of a set of features in the study of another phenomenon, lies in the nature of movement on the basis of which the level of the subject position on a status feature is determined. In fact, most other anthropological characteristics can be precisely, on the basis of common physical measures, reliably and objectively measured or estimated on sufficiently stringent metric variables, simply because they are the result of efficiency of natural (physiological, biochemical and other biological) systems.

The main significance and basic mode of operation of these systems is, of course, regular and universal for every human being. The individual differences, which undoubtedly exist, are a consequence of either genetically conditioned differences or effects of external stimulus (such as, learning or training), or a consequence of a disease or some other endogenously or exogenously induced disorders.

However, the criteria for determining social status characteristics are, as a rule, extra individual and based on the socio-economic structure of the society, the collective value system, particularly on the structure and efficiency of the institutional mechanisms for regulating social relations.

Therefore, the determination of the position of an individual in the social field is hard to be carried out by reasonable estimate, and even harder by exact measurement. Status variables are typically of an actuarial type, the result of conventions that do not necessarily have to be guided by their real sociological significance, and the results are most commonly found on non-metric scales.

Perhaps the biggest problem in the study of social status, the problem of metric properties of status variables, arises from this. Simple operations of encoding, which are, at best, products of an ordinal variable, are usually performed over the information that is typically of an actuarial type. It is not uncommon that because of the nature of the problem of the observed feature, a certain status variable lies on a semi-ordinal scale. The best example is the variable that is often used to assess the social status of a family and which is related to the education of one or both parents.

However, there are other sources of methodological difficulties associated with examining the social status, and, therefore, with construction of any verified theory of social differentiation, or social stratification, or class differences, or social distinction at all.

Consideration of these theories and the way of conducting studies of social status, show that the main methodological problems in this area are as follows:

1) The construction or selection of models on the basis of which the universe of status variables is defined;

2) The definition of the population to which the results of any research or formulation of any theory could relate, and the method of selecting a sample from the population;

3) Determination of manifest status categories and development of measurement instruments for their regulation or measurement;

4) Adequacy of models, methods and techniques for data analysis and hypothesis testing.

Unlike many other anthropological phenomena for which during the development of the appropriate sciences, a number of structural models which differ from each other, depending on the author's scientific orientation, have been constructed; at the moment there is only one model of the social status structure, it was developed by a group of researchers of the Institute of Sociology and Philosophy, University of Ljubljana. This model, which, in its first international presentation at the 
International Congress of Sociologists in Toronto in 1974, received the most favorable reviews of sociologists of both eastern and western countries and served as the basis for this study.

The results presented in Table 1 show that there is a statistically significant multiple correlation between cognitive dimensions, conative variables and social factors and academic achievement in the Serbian language course (RO $=.41)$.

Detailed analysis of the numerical values of the regression analysis, or partial correlations and beta coefficients, shows that the association of cognitive abilities, personality traits and social attitudes with school grades in the Serbian language course is significant and defined by:

- a single cognitive factor, or a factor of perceptual reasoning;

- a conative factor by which the regulator of attack is estimated;

- the social status of the mother's education, mother's knowledge of a foreign language, maternal grandfather's education and student's overall academic achievement.

From the above, it necessarily follows that technically qualified people who have a developed function of the input processor, or better perceptual reasoning, greater control of attack responses, a solid education of mother, her knowledge of a foreign language, maternal grandfather's better education, and better overall academic achievement, are more predisposed to learning mother tongue, which is fully justified in terms of a learning theory.

Table 1. Regression of the grade in Serbian language and predictor variables.

\begin{tabular}{llllll}
\hline & $\mathbf{R}$ & Partial R & Beta & $\mathbf{t}$ & Sig. \\
\hline AL-4 & .04 & .04 & .05 & .68 & 49 \\
IT-1 & -.13 & -.14 & -.15 & -2.14 & .03 \\
S-1 & .05 & .05 & .06 & .79 & .42 \\
EPSILON & -.03 & -.04 & -.04 & -.56 & .57 \\
CHI & .06 & .07 &, 11 & 1.06 & .28 \\
ALPHA & -.06 & -.06 & -.10 & -.95 & .34 \\
SIGMA & -.18 & -.19 & -.22 & -2.88 & .00 \\
DELTA & .05 & .05 & .07 & .78 & .43 \\
ETA & .03 & .04 & .07 & .57 & .56 \\
EFA & .00 & .00 & .00 & .00 & .99 \\
EMO & .14 & .15 & .20 & 2.27 & .02 \\
FOL & .02 & .02 & .03 & .40 & .68 \\
FOLFA & .03 & .03 & .04 & .50 & .61 \\
FOLMO & -.14 & -.15 & -.19 & -2.19 & .02 \\
SCHOOL & .09 & .10 & .11 & 1.53 & .12 \\
QFA & -.02 & -.02 & -.03 & -.31 & .75 \\
QMO & .00 & .00 & .00 & .03 & .97 \\
EPGFA & .10 & .11 & .16 & 1.58 & .11 \\
EMGFA & -.20 & -.22 & -.32 & -3.24 & .00 \\
ACACH & .12 & .13 & .13 & 1.87 & .05 \\
SPACH & -.04 & -.04 & -.05 & -.69 & .48 \\
PL & -.02 & -.02 & -.04 & -.38 & .70 \\
PLFA & .07 & .08 & .15 & 1.20 & .23 \\
PLMO & -.00 & -.00 & -.00 & -.05 & .95 \\
\hline RO & DELTA & $\mathrm{df} 1$ & $\mathrm{df} 2$ & $\mathrm{~F}$ & Sig. \\
.41 & .17 & 24 & 200 & 1.78 & .01 \\
\hline
\end{tabular}

\section{Conclusion}

The study was conducted in order to determine the influence of cognitive dimensions, conative characteristics and social status on school grades in the native language course of the students of a secondary school of chemical technology.

For this purpose, 224 students of a secondary school of chemical technology were included in the sample. This research has provided unequivocal evidence that the structure of cognitive abilities is of a hierarchical type, with the general cognitive factor at the top and three primary factors of cognitive abilities below it. These three factors are related to the effectiveness of the perceptual processor (or perceptual reasoning), the effectiveness of the parallel processor (or the ability to identify relations and correlates) and the effectiveness of the serial processor (or symbolic reasoning).

To evaluate the effectiveness of the input processor, or perceptual reasoning, IT-1 test was chosen. To evaluate the effectiveness of the serial processor, or symbolic reasoning, AL-4 test was selected. To evaluate the effectiveness of the parallel processor, or identification of relations and correlates, S-1 test was chosen.

For the assessment of conative characteristics, CON6 measuring instrument was selected by means of which the following conative regulators were evaluated: regulator of activity, regulator of organic functions, regulator of defense reactions, regulator of attack reactions, system for coordination of regulatory functions, system for integration of regulatory functions and system for excitation and inhibition.

To evaluate social status, the model constructed by Saksida and Momirović was applied. INST2 and questionnaire SSMIN were used in this research.

For the evaluation of students' academic achievements, their school grades were used. The academic achievements were checked by means of knowledge tests as indicators of the acquired knowledge and skills.

The students' academic achievements are presented in this study by a traditional for this country way of academic evaluation, i.e. numerical grades of 1 to 5 at the end of the school year (the so-called final grade) for all educational-scientific areas: languages - mother tongue and foreign languages.

All the data in this study were processed at the Multidisciplinary Research Center, Faculty of Sport and Physical Education, University of Pristina, through the system of data processing software programs DRSOFT developed by Popovic, D. (1980, 1993) and Momirovic, K. \& Popovic, D . (2003).

The algorithms and programs implemented within this study are presented in full, and the results of these programs are analyzed.

The results presented in Table 1 show that there is a statistically significant multiple correlation between cognitive dimensions, conative variables and social factors and academic achievement in Serbian language $(\mathrm{RO}=.41)$.

The detailed analysis of the numerical values of regression 
analysis, or partial correlations and beta coefficients, shows that the association of cognitive abilities, personality characteristics and social attitudes with the school grades in Serbian language is significant and defined by

- a single cognitive factor, or factor of perceptual reasoning;

- $\quad$ conative factor which estimates the regulator of attacks;

- $\quad$ social status, mother's education, mother's knowledge of a foreign language, maternal grandfather's education and student's overall academic achievement.

From the above, it necessarily follows that technically qualified people have a developed function of the input processor, that is, better perceptual reasoning, greater control of attack reactions, mother's solid education, her knowledge of a foreign language, maternal grandfather's better education and student's better overall academic achievement, and they are more predisposed to learning their mother tongue, which is fully justified in terms of a learning theory.

\section{References}

[1] Boli, E. (1996). The structure of intellectual and musical abilities and personal traits of girls involved in standard and Latin American dance, Master's thesis, Pristina, University of Pristina, Faculty of Physical Education.

[2] Boli, E. (2011). The structure of anthropological dimensions of male and female dancers and development of procedures for their evaluation and monitoring. (Monograph), Leposavic, University in Pristina, Multidisciplinary Research Center of the Faculty of Sport and Physical Education.

[3] Boli, E., Popovic, D., Popovic, J. (2012) Structure of intellectual abilities of dancers, International scientific journal Kinesmetrics, 1 (91-105).

[4] Boli, E., Popovic, D., Popovic, J. (2012) The structure of personality characteristics of dancers, International scientific journal Kinesmetrics, 1 (161-177).

[5] Boli, E., Popovic, D., Popovic, J. (2012). The structure of the social status of dancers, International scientific journal Kinesmetrics, 1 (193-221).

[6] Boli, E., Popović, D., Popović, J. (2012). Differences in the level of cognitive abilities of male and female dancers, International scientific journal Kinesmetrics, 1 (107-119).

[7] Hadzigalic, S., Bogdanovic, M., Tenjovic, L., Wolf, B. (1994). About some characteristics of Mahalanobis spaces. Proceedings, 8 Section for classifications of the Union of Statistics Societies of Yugoslavia, Belgrade: Federal Institute for Statistics, (pp.99-132).
[8] Momirovic, D., Wolf, B., Popovic, D. (1999) Introduction to the theory of measurement and internal metric properties of composite measurement instruments (textbook), University of Pristina, Pristina, Faculty of Physical Culture.

[9] Momirovic, K., Popovic, D. (2003) Construction and application of taxonomic neural networks, Multidisciplinary Research Center, Faculty of Physical Education

[10] Mulaik, S. A. (1972). The foundations of factor analysis. New York: McGraw-Hill.

[11] Popovic, D. (1980) Research Methodology in Physical Education, University of Nis, Scientific Youth, Nis.

[12] Popovic, D., Antic, K., Stankovic, V., Petkovic, V., Stankovic, S. (1989) The procedures for objectification of estimating the effectiveness in performing the judo techniques. Scientific Youth, 21(1-2), 83-89.

[13] Popovic, D., Kocic, J., Boli, E., Stankovic, V. (1995) Conative characteristics of female dancers. International Congress "Images of Sport in the World", 75th Anniversary of the German Sport University, Abstract Volume, (pp. 96), Open Forum, Cologne, Germany.

[14] Popovic, D., Petrovic, J., Boli, E., Stankovic, V. (1995) The personality structure of female dancers. 3rd International Congress on Physical Education and Sport, Exercise \& Society supplement issue No. 11 (pp. 196), Komotini, Greece.

[15] Popovic, D., Stankovic, V., Kulic, R., Grigoropulos, P. (1996) The personality structure of handball players. 4th International Congress on Physical Education and Sport, Exercise \& Society supplement issue No. 15 (pp. 164), Komotini, Greece.

[16] Popovic, D. (2005). GUTTMAN, Programs for analysis of metrical characteristics of composite measurement instruments in Savic, Z.: Influence of situational training on transformation of some anthropological dimensions in selected footballers (doctoral thesis) Leposavic, Faculty of Physical Culture.

[17] Popovic, D. (1988) Methods of factor analysis application for determining morphological types. 4rd International symposium on the methodology of mathematical modelling, Varna, Bulgaria.

[18] Popovic, D. (1991) Research Methodology in Physical Education (textbook), University of Nis, Scientific Youth, Nis.

[19] Popovic, D. (1992) Methodology of research in physical education, Athens, Greece.

[20] Popovic, D. (1993)Programs and subprograms for the analysis of quantitative modifications (textbook), University of Pristina, Faculty of Physical Education, Multidisciplinary Research Center, Pristina.

[21] Popovic, D.(1993) Determining the structure of psychosomatic dimensions in martial arts and developing methods for their assessment and monitoring (monograph), University of Pristina, Faculty of Physical Culture, Pristina. 